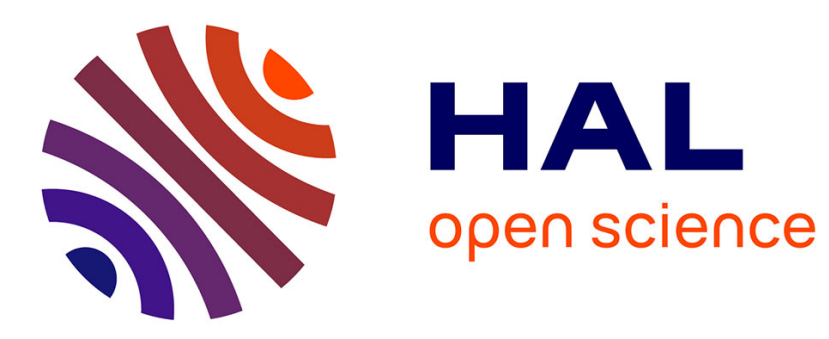

\title{
Weighted Improper Colouring
}

Julio Araujo, Jean-Claude Bermond, Frédéric Giroire, Frédéric Havet, Dorian Mazauric, Remigiusz Modrzejewski

\section{To cite this version:}

Julio Araujo, Jean-Claude Bermond, Frédéric Giroire, Frédéric Havet, Dorian Mazauric, et al.. Weighted Improper Colouring. 22th International Workshop, IWOCA 2011, Jul 2011, Victoria, Canada. inria-00635882

\section{HAL Id: inria-00635882 https://hal.inria.fr/inria-00635882}

Submitted on 26 Oct 2011

HAL is a multi-disciplinary open access archive for the deposit and dissemination of scientific research documents, whether they are published or not. The documents may come from teaching and research institutions in France or abroad, or from public or private research centers.
L'archive ouverte pluridisciplinaire HAL, est destinée au dépôt et à la diffusion de documents scientifiques de niveau recherche, publiés ou non, émanant des établissements d'enseignement et de recherche français ou étrangers, des laboratoires publics ou privés. 


\title{
Weighted Improper Colouring *
}

\author{
Julio Araujo ${ }^{1,2}$, Jean-Claude Bermond ${ }^{1}$, Frédéric Giroire ${ }^{1}$, Frédéric Havet ${ }^{1}$, Dorian Mazauric ${ }^{1}$, and \\ Remigiusz Modrzejewski ${ }^{1}$ \\ 1 Mascotte, joint project I3S(CNRS/Univ. de Nice)/INRIA, France \\ 2 ParGO Research Group - Universidade Federal do Ceará - UFC, Brazil
}

\begin{abstract}
In this paper, we study a colouring problem motivated by a practical frequency assignment problem and up to our best knowledge new. In wireless networks, a node interferes with the other nodes the level of interference depending on numerous parameters: distance between the nodes, geographical topography, obstacles, etc. We model this with a weighted graph $G$ where the weights on the edges represent the noise (interference) between the two end-nodes. The total interference in a node is then the sum of all the noises of the nodes emitting on the same frequency. A weighted $t$-improper $k$-colouring of $G$ is a $k$-colouring of the nodes of $G$ (assignment of $k$ frequencies) such that the interference at each node does not exceed some threshold $t$. The Weighted Improper Colouring problem, that we consider here consists in determining the weighted $t$-improper chromatic number defined as the minimum integer $k$ such that $G$ admits a weighted $t$-improper $k$-colouring. We also consider the dual problem, denoted the Threshold Improper Colouring problem, where given a number $k$ of colours (frequencies) we want to determine the minimum real $t$ such that $G$ admits a weighted $t$-improper $k$-colouring. We show that both problems are NP-hard and first present general upper bounds; in particular we show a generalisation of Lovász's Theorem for the weighted $t$-improper chromatic number. We then show how to transform an instance of the Threshold Improper Colouring problem into another equivalent one where the weights are either 1 or $M$, for a sufficient big value $M$. Motivated by the original application, we study a special interference model on various grids (square, triangular, hexagonal) where a node produces a noise of intensity 1 for its neighbours and a noise of intensity $1 / 2$ for the nodes that are at distance 2 . Consequently, the problem consists of determining the weighted $t$-improper chromatic number when $G$ is the square of a grid and the weights of the edges are 1, if their end nodes are adjacent in the grid, and 1/2 otherwise. Finally, we model the problem using linear integer programming, propose and test heuristic and exact Branch-and-Bound algorithms on random cell-like graphs, namely the Poisson-Voronoi tessellations.
\end{abstract}

\section{Introduction}

Let $G=(V, E)$ be a graph. A $k$-colouring of $G$ is a function $c: V \rightarrow\{1, \ldots, k\}$. The colouring $c$ is proper if $u v \in E$ implies $c(u) \neq c(v)$. The chromatic number of $G$, denoted by $\chi(G)$, is the minimum integer $k$ such that $G$ admits a proper $k$-colouring. The goal of the VERTEX COLOURING problem is to determine $\chi(G)$ for a given graph $G$. It is a well-known NP-hard problem [11].

A $k$-colouring $c$ is $l$-improper if $|\{v \in N(u) \mid c(v)=c(u)\}| \leq l$ for all $u \in V$. Given a non-negative integer $l$, the $l$-improper chromatic number of a graph $G$, denoted by $\chi_{l}(G)$, is the minimum integer $k$ such that $G$ has an $l$-improper $k$-colouring. For given graph $G$ and integer $l$, the IMPROPER COLOURING problem consists in determining $\chi_{l}(G)[6,14]$ and is also NP-hard. Indeed, if $l=0$, observe that $\chi_{0}(G)=\chi(G)$. Consequently, VERTEX COLOURING is a particular case of IMPROPER COLOURING.

In this work we define and study a new variation of the improper colouring problem for edge-weighted graphs. Given an edge-weighted graph $G=(V, E, w), w: E \rightarrow \mathbb{R}_{+}^{*}$, a threshold $t \in \mathbb{R}_{+}$, and a colouring $c$, we note the interference of a vertex $w$ in this colouring as:

$$
I_{u}(G, w, c)=\sum_{\{v \in N(u) \mid c(v)=c(u)\}} w(u, v) .
$$

We say that $c$ is a weighted t-improper $k$-colouring of $G$ if $c$ is a $k$-colouring of $G$ such that $I_{u}(G, w, c) \leq t$, for all $u \in V$.

\footnotetext{
* This work was partially supported by région PACA, ANR Blanc AGAPE and ANR International Taiwan GRATEL.
} 
Given a threshold $t \in \mathbb{R}_{+}^{*}$, the minimum integer $k$ such that the graph $G$ admits a weighted $t$-improper $k$ colouring is the weighted $t$-improper chromatic number of $G$, denoted by $\chi_{t}(G, w)$. Given an edge-weighted graph $G=(V, E, w)$ and a threshold $t \in \mathbb{R}_{+}^{*}$, determining $\chi_{t}(G, w)$ is the goal of the WEIGHTED IMPROPER COLOURING problem. Note that if $t=0$ then $\chi_{0}(G, w)=\chi(G)$, and if $w(e)=1$ for all $e \in E$, then $\chi_{l}(G, w)=\chi_{l}(G)$ for any positive integer $l$. Therefore, the WeIGHTEd IMPROPER Colouring problem is clearly NP-hard since it generalises VerTEX COLOURING and IMPROPER COLOURING.

On the other hand, we define the minimum k-threshold of $G$, denoted by $\omega_{k}(G, w)$ as the minimum real $t$ such that $G$ admits a weighted $t$-improper $k$-colouring. Then, for a given edge-weighted graph $G=(V, E, w)$ and a positive integer $k$, the THRESHOLD IMPROPER COLOURING problem consists of determining $\omega_{k}(G, w)$.

Motivation. Our initial motivation to these problems was the design of satellite antennas for multi-spot MFTDMA satellites [2]. In this technology, satellites transmit signals to areas on the ground called spots. These spots form a grid-like structure which is modelled by an hexagonal cell graph. To each spot is assigned a radio channel or colour. Spots are interfering with other spots having the same channel and a spot can use a colour only if the interference level does not exceed a given threshold $t$. The level of interference between two spots depends on their distance. The authors of [2] introduced a factor of mitigation $\gamma$ and the interferences of remote spots are reduced by a factor $1-\gamma$. When the interference level is too low, the nodes are considered to not interfere anymore. Considering such types of interferences, where nodes at distance at most $i$ interfere, leads to the study of the $i$-th power of the graph modelling the network and a case of special interest is the power of grid graphs (see Section 3).

Related Work. Our problems are particular cases of the FREQUenCy ASSIGNMENT PRoblem (FAP). FAP has several variations that were already studied in the literature (see [1] for a survey). In most of these variations, the main constraint to be satisfied is that if two vertices (mobile phones, antennas, spots, etc.) are close, then the difference between the frequencies that are assigned to them must be greater than some function that usually depends on their distance.

There is a strong relationship between most of these variations and the $L\left(p_{1}, \ldots, p_{d}\right)$-LABELLING PROBLEM [15]. In this problem, the goal is to find a colouring of the vertices of a given graph $G$ in such a way that the difference between the colours assigned to vertices at distance $i$ must be at least $p_{i}$, for every $i=1, \ldots, d$.

For some other variations, for each non-satisfied interference constraint a penalty must be paid. In particular, the goal of the Minimum InTERfEREnCE Assignment Problem (MI-FAP) is to minimise the total penalties that must be paid, when the number of frequencies to be assigned is given. This problem can also be studied for only co-channel interferences, in which the penalties are applied only if the two vertices have the same frequency. However, MI-FAP under these constraints does not correspond to WEIGHTED IMPROPER COLOURING, because we consider the co-channel interference, i.e. penalties, just between each vertex and its neighbourhood.

The two closest related works we found in the literature are [13] and [7]. However, they both apply penalties over co-channel interference, but also to the adjacent channel interference, i.e. when the colours of adjacent vertices differ by one unit. Moreover, their results are not similar to ours. In [13], they propose an enumerative algorithm for the problem, while in [7] a Branch-and-Cut method is proposed and applied over some instances.

\section{Results.}

In this article, we study both parameters $\chi_{t}(G, w)$ and $\omega_{k}(G, w)$. We first show that ThreSHOLD IMPROPER COLOURING is NP-hard. Then we present general upper bounds; in particular we show a generalisation of Lovász' Theorem for $\chi_{t}(G, w)$. We then show how to transform an instance into an equivalent one where the weights are either 1 or $M$, for a sufficient big value $M$.

Motivated by the original application, we study a special interference model on various grids (square, triangular, hexagonal) where a node produces a noise of intensity 1 for its neighbours and a noise of intensity $1 / 2$ for the nodes that are at distance 2 . Consequently, the problem consists of determining $\chi_{t}(G, w)$ and $\omega_{k}(G, w)$, when $G$ is the square of a grid and the weights of the edges are 1, if their end nodes are adjacent in the grid, and $1 / 2$ otherwise.

Finally, we propose a heuristic and a Branch-and-Bound algorithm to solve the THRESHOLD IMPROPER COLOURING for general graphs. We compare them to an integer programming formulation on 


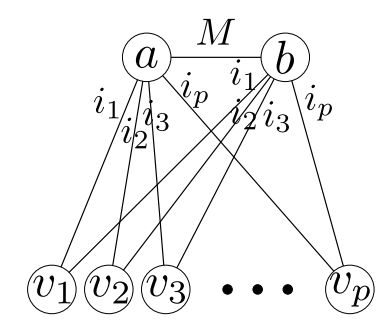

Fig. 1. Construction of $G(I, t)$ from an instance $(I, t)$ of the PARTition Problem.

random cell-like graphs, namely Voronoi diagrams of random points of the plan. These graphs are classically used in the literature to model telecommunication networks $[4,8,9]$.

\section{General Results}

In this section, we present some results for WEIGHTED IMPROPER COLOURING and THRESHOLD IMPROPER COLOURING for general graphs and general interference models.

\subsection{NP-Completeness of THRESHOLD IMPROPER COLOURING}

In this section, we prove that the decision problem associated to THRESHOLD IMPROPER COLOURING is NP-complete already for $k=2$.

Theorem 1. The following problem is NP-complete.

Instance: An edge-weighted graph $G=(V, E, w), w: E \rightarrow \mathbb{R}_{+}^{*}$, a threshold $t \in \mathbb{R}_{+}$. Question: Does $G$ have a weighted t-improper 2-colouring?

Proof. Given a 2-colouring $c$ of $G$, one can test in $O(|E|)$-time if $c$ is weighted $t$-improper by just checking, for each vertex $v$, if $I_{v}(G, w, c) \leq t$. Consequently, this problem is in NP.

Now we reduce the PARTITION problem [11] which is NP-complete, to it. In the PARTITION problem, given a set of $p$ positive integers $I=\left\{i_{1}, \ldots, i_{p}\right\}$ and a threshold $t$, we want to decide if there is a partition of the elements of $I$ into two sets $A$ and $B$ such that $\sum_{i_{a} \in A} i_{a} \leq t$ and $\sum_{i_{b} \in B} i_{b} \leq t$. We consider that $i_{j} \leq t$, for all $j \in\{1, \ldots, p\}$, and that $t \leq \sum_{j=1}^{p} i_{j}$, otherwise the answer for this problem is trivially no and yes, respectively.

Given an instance $(I, t)$ of the PARTition Problem, let $G(I, t)$ be a graph whose vertex set is $V(G(I, t))=$ $\left\{v_{j} \mid j \in\{1, \ldots, p\}\right\} \cup\{a, b\}$ and whose edge set is $E(G(I, t))=\{(a, b)\} \cup\left\{\left(a, v_{j}\right),\left(b, v_{j}\right) \mid j \in\{1, \ldots, p\}\right\}$ (see Figure 1). Define $M=1+\sum_{j=1}^{p} i_{j}$. Let $w: E(G(I, t)) \rightarrow\left\{i_{1}, \ldots, i_{p}, M\right\}$ be a weight function for the edges of $G(I, t)$ defined in the following way: $w(a, b)=M$ and $w\left(a, v_{j}\right)=w\left(b, v_{j}\right)=i_{j}$, for every $j \in\{1, \ldots p\}$.

We claim that $(I, t)$ is a yes answer for the PARTITION Problem if, and only if, $G(I, t)$ admits a weighted $t$-improper 2-colouring.

If $(I, t)$ is a yes instance, let $(A, B)$ be a partitioning such that $\sum_{i_{a} \in A} i_{a} \leq t$ and $\sum_{i_{b} \in B} i_{b} \leq t$. We claim that the following colouring $c$ is a weighted $t$-improper 2-colouring of $G(I, t)$ :

$$
c(v)= \begin{cases}1 & \text { if } v \in\{a\} \cup\left\{v_{j} \mid i_{j} \in A\right\} \\ 2 & \text { otherwise }\end{cases}
$$

To verify this fact, observe that $I_{a}(G, w, c)=\sum_{i_{j} \in A} i_{j} \leq t$, that $I_{b}(G, w, c)=\sum_{i_{j} \in B} i_{j} \leq t$ and that $I_{v_{j}}(G, w, c)=$ $i_{j} \leq t$, for each $j \in\{1 \ldots, p\}$.

Conversely, consider that $G(I, t)$ admits a weighted $t$-improper 2-colouring $c$. Remark that $a$ and $b$ must receive different colours since the weight of the edge $(a, b)$ is $M>t$. Thus, assume that $c(a)=1$ and that $c(b)=2$. Let $A$ be the subset of integers $i_{j}, j \in\{1, \ldots, p\}$, such that $c\left(v_{j}\right)=1$ and $B=I \backslash A=\left\{i_{j} \mid c\left(v_{j}\right)=\right.$ $2\}$. Observe that the sum of elements in $A$ (resp. $B$ ) is equal to $I_{a}(G, w, c)$ (resp. $I_{b}(G, w, c)$ ) and they are both smaller or equal to $t$, since $c$ is a weighted $t$-improper 2-colouring. 


\subsection{Bounds}

Upper bound for WEIGHTED IMPROPER COLOURING It is a folklore result $\chi(G) \leq \Delta(G)+1$, for any graph $G$. Lovász [12] extended this result for IMPROPER COLOURING problem. He proved that $\chi_{l}(G) \leq$ $\left\lceil\frac{\Delta(G)+1}{l+1}\right\rceil$. In what follows, we show an extension of these results to WEIGHTED IMPROPER COLOURING.

Given an edge-weighted graph $G=(V, E, w), w: E \rightarrow \mathbb{R}_{+}^{*}$, and $v \in V$, let $d_{w}(v)=\sum_{u \in N(v)} w(u, v)$. Denote by $\Delta(G, w)=\max _{v \in V} d_{w}(v)$. Given a $k$-colouring $c: V \rightarrow\{1, \ldots, k\}$ of $G$, we denote $d_{w, c}^{i}(v)=$ $\sum_{\{u \in N(v) \mid c(u)=i\}} w(u, v)$, for every vertex $v \in V$ and colour $i=1, \ldots, k$. Note that $d_{w, c}^{c(v)}(v)=I_{v}(G, w, c)$. Finally, we denote $\operatorname{gcd}(w)$ the greatest common divisor of the weights of $w$. We use here the generalisation of the gcd to non-integer numbers (e.g. in $\mathbb{Q}$ ) where a number $x$ is said to divide a number $y$ if the fraction $y / x$ is an integer. The important property of $\operatorname{gcd}(w)$ is that the difference between two interferences is a multiple of $\operatorname{gcd}(w)$; in particular, if for two vertices $v$ and $u, d_{w, c}^{i}(v)>d_{w, c}^{j}(u)$, then $d_{w, c}^{i}(v) \geq d_{w, c}^{j}(u)+\operatorname{gcd}(w)$.

If $t$ is not a multiple of the $\operatorname{gcd}(w)$, that is, there exists an integer $a \in \mathbb{Z}$ such that $a \operatorname{gcd}(w)<t<$ $(a+1) \operatorname{gcd}(w)$, then $\chi_{t}^{w}(G)=\chi_{a \operatorname{gcd}(w)}^{w}(G)$.

Theorem 2. Given an edge-weighted graph $G=(V, E, w), w: E \rightarrow \mathbb{Q}_{+}^{*}$, and a threshold $t$ multiple of $\operatorname{gcd}(w)$, then the following inequality holds:

$$
\chi_{t}(G, w) \leq\left\lceil\frac{\Delta(G, w)+\operatorname{gcd}(w)}{t+\operatorname{gcd}(w)}\right\rceil .
$$

Proof. We say that a $k$-colouring $c$ of $G$ is well-balanced if $c$ satisfies the following property:

Property 1. For any vertex $v \in V, I_{v}(G, w, c) \leq d_{w, c}^{j}(v)$, for every $j=1, \ldots, k$.

If $k=1$ there is nothing to prove. Then, we prove that for any $k \geq 2$, there exists a well-balanced $k$ colouring of $G$. To prove this fact one may just colour $G$ arbitrarily with $k$ colours and then repeat the following procedure: if there exists a vertex $v$ coloured $i$ and a colour $j$ such that $d_{w, c}^{i}(v)>d_{w, c}^{j}(v)$, then recolour $v$ with colour $j$. Observe that this procedure neither increases (we just move a vertex from one colour to another) nor decreases (a vertex without neighbour on its colour is never moved) the number of colours within this process. Let $W$ be the sum of the weights of the edges having the same colour in their endpoints. In this transformation, $W$ has increased by $d_{w, c}^{j}(v)$ (edges that previously had colours $i$ and $j$ in their endpoints), but decreased by $d_{w, c}^{i}(v)$ (edges that previously had colour $i$ in both of their endpoints). So, $W$ has decreased by $d_{w, c}^{i}(v)-d_{w, c}^{j}(v) \geq \operatorname{gcd}(w)$. As $W \leq|E| \max _{e \in E} w(e)$ is finite, this procedure finishes and produces a well-balanced $k$-colouring of $G$.

Observe that in any well-balanced $k$-colouring $c$ of a graph $G$, the following holds:

$$
d_{w}(v)=\sum_{u \in N(v)} w(u, v) \geq k d_{w, c}^{c(v)}(v) .
$$

Let $k^{*}=\left\lceil\frac{\Delta(G, w)+\operatorname{gcd}(w)}{t+\operatorname{gcd}(w)}\right\rceil \geq 2$ and $c^{*}$ be a well-balanced $k^{*}$-colouring of $G$. We claim that $c^{*}$ is a weighted $t$-improper $k^{*}$-colouring of $G$.

By contradiction, suppose that there is a vertex $v$ in $G$ such that $c^{*}(v)=i$ and that $d_{w, c}^{i}(v)>t$. Since $c^{*}$ is well-balanced, $d_{w, c}^{j}(v)>t$, for all $j=1, \ldots, k^{*}$. By the definition of $\operatorname{gcd}(w)$ and as $t$ is a multiple of $\operatorname{gcd}(w)$, it leads to $d_{w, c}^{j}(v) \geq t+\operatorname{gcd}(w)$ for all $j=1, \ldots, k^{*}$. Combining this inequality with Inequality (1), we obtain:

$$
\Delta(G, w) \geq d_{w}(v) \geq k^{*}(t+\operatorname{gcd}(w))
$$

giving

$$
\Delta(G, w) \geq \Delta(G, w)+\operatorname{gcd}(w)
$$

a contradiction. The result follows. 


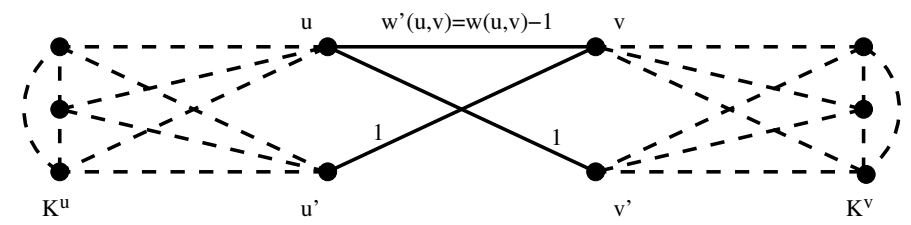

Fig. 2. Construction of $G^{i+1}$ from $G^{i}$ using edge $(u, v)$ with $k=4$. Dashed edges represent edges with infinite weights.

Note that when all weights are equal to one, we obtain the bound for the improper colouring derived in [12].

Brooks [5] proved that for a connected graph $G, \chi(G)=\Delta(G)+1$ if, and only if, $G$ is complete or an odd cycle. One could wonder for which edge-weighted graphs the bound we provide is tight. However, Correa et al. [6] already showed that it is NP-complete to determine if the improper chromatic number of a graph $G$ attains the upper bound of Lovász, which is a particular case of WEIGHTED IMPROPER COLOURING and the bound we provided.

Upper bound for Threshold Improper Colouring Let $G=(V, E, w), w: E \rightarrow \mathbb{R}_{+}^{*}$, be an edgeweighted graph and $k$ be a positive integer. Observe that, for the minimum $k$-threshold of $G$,

$$
\omega_{k}(G, w) \leq \Delta(G, w) \leq \sum_{e \in E(G)} w(e) .
$$

In what follows, we improve this trivial upper bound.

Let $V^{\prime}=\{u \in V, d(u) \geq k\}$ be the set of vertices with degree at least $k$. Set $G^{\prime}=G-V^{\prime}$.

Lemma 1. $\omega_{k}(G, w)=\omega_{k}\left(G^{\prime}, w\right)$

Proof. If there is a weighted $t$-improper $k$-colouring of $G^{\prime}$, then it is easy to get a weighted $t$-improper $k$-colouring of $G$ choosing, for each vertex $u \in V \backslash V^{\prime}$, a colour different from the colours of its neighbours. It is always possible because $d(u) \leq k-1$.

Conversely, if there is a weighted $t$-improper $k$-colouring of $G$, then there is a weighted $t$-improper $k$-colouring of $G^{\prime}$ by choosing, for every $v \in V^{\prime}, c_{G^{\prime}}(v)=c_{G}(v)$.

For the rest of the section, we only consider edge-weighted graphs with minimum degree at least $k$. For each $v \in V$, let $E_{\min }^{k-1}(v)$ be the set of $d(v)-(k-1)$ least weighted edges incident to $v$.

Theorem 3. Let $G=(V, E, w), w: E \rightarrow \mathbb{R}_{+}^{*}$, be an edge-weighted graph and $k$ be a positive integer. Then,

$$
\omega_{k}(G, w) \leq \max _{v \in V} w\left(E_{\min }^{k-1}(v)\right)
$$

where $w\left(E_{\min }^{k-1}(v)\right)=\sum_{e \in E_{\min }^{k-1}(v)} w(e)$.

Proof. Let $G_{\min }^{k-1}=G\left[E \backslash\left\{\bigcup_{v \in V} E_{\min }^{k-1}(v)\right\}\right]$. Observe that the maximum degree of a vertex in $G_{\min }^{k-1} \leq k-1$. Consequently, $G_{\min }^{k-1}$ admits a proper $k$-colouring $c$ of its vertices.

Observe that the maximum interference of a vertex $v$ in $G$ when $G$ is coloured by the colouring $c$ is at $\operatorname{most}_{\max } w \in\left(E_{\min }^{k-1}(v)\right)$ and the result follows.

\subsection{Transformation}

In this section, we prove that the THRESHOLD IMPROPER COLOURING problem can be transformed into a problem mixing proper and improper colouring. More precisely, we prove the following:

Theorem 4. Let $G_{0}=\left(V_{0}, E_{0}, w_{0}\right)$ be an edge-weighted graph such that, for every $e \in E, w(e) \in \mathbb{Z}_{+}^{*}$, and $k$ be a positive integer. We can construct a graph $G^{*}=\left(V^{*}, E^{*}, w^{*}\right)$ such that $w^{*}(e) \in\{1, M\}$ for any $e \in E\left(G^{*}\right)$, satisfying $\omega_{k}\left(G_{0}, w_{0}\right)=\omega_{k}\left(G^{*}, w^{*}\right)$, where $M=1+\sum_{e \in E(G)} w_{0}(e)$. 
Proof. Consider the function $f(G, w)=\sum_{\{e \in E(G) \mid w(e) \neq M\}}(w(e)-1)$.

If $f(G, w)=0$, all edges have weight either 1 or M and $G$ has the desired property. In this case, $G^{*}=G$. Otherwise, we construct a graph $G^{\prime}$ and a function $w^{\prime}$ such that $\omega_{k}\left(G^{\prime}, w^{\prime}\right)=\omega_{k}(G, w)$, but $f\left(G^{\prime}, w^{\prime}\right)=$ $f(G, w)-1$. By repeating this operation $f\left(G_{0}, w_{0}\right)$ times we get the required graph $G^{*}$.

In case $f(G, w)>0$, there exists an edge $e=(u, v) \in E(G)$ such that $2 \leq w(e)<M$. $G^{\prime}$ is obtained from $G$ by adding two complete graphs on $k-1$ vertices $K^{u}$ and $K^{v}$ and two new vertices $u^{\prime}$ and $v^{\prime}$. We join $u$ and $u^{\prime}$ to all the vertices of $K^{u}$ and $v$ and $v^{\prime}$ to all the vertices of $K^{v}$. We assign weight $M$ to all these edges. Note that, $u$ and $u^{\prime}\left(v\right.$ and $v^{\prime}$ ) always have the same colour, namely the remaining colour not used in $K^{u}$ (resp. $K^{v}$ ).

We also add two edges $u v^{\prime}$ and $u^{\prime} v$ both of weight 1 . The edges of $G$ keep their weight in $G^{\prime}$, except the edge $e=u v$ whose weight is decreased by one unit, i.e., $w^{\prime}(e)=w(e)-1$. Thus, $f\left(G^{\prime}\right)=f(G)-1$ as we added only edges of weights 1 and $M$ and we decreased the weight of $e$ by one unit.

Now consider a weighted $t$-improper $k$-colouring $c$ of $G$. We produce a weighted $t$-improper $k$-colouring $c^{\prime}$ to colour $G^{\prime}$ as follows: we keep the colours of all the vertices in $G$, we assign to $u^{\prime}\left(v^{\prime}\right)$ the same colour as $u$ (resp., $v$ ), and we assign to $K^{u}\left(K^{v}\right)$ the $k-1$ colours different from the one used in $u$ (resp. $v$ ).

Conversely, from any weighted improper $k$-colouring $c^{\prime}$ of $G^{\prime}$, we get a weighted improper $k$-colouring $c$ of $G$ by just keeping the colours of the vertices that belong to $G$.

For such colourings $c$ and $c^{\prime}$ we have that $I_{x}(G, w, c)=I_{x}\left(G^{\prime}, w^{\prime}, c^{\prime}\right)$, for any vertex $x$ of $G$ different from $u$ and $v$. For $x \in K^{u} \cup K^{v}, I_{x}\left(G^{\prime}, w^{\prime}, c^{\prime}\right)=0$. The neighbours of $u$ with the same colour as $u$ in $G^{\prime}$ are the same as in $G$, except possibly $v^{\prime}$ which has the same colour of $u$ if, and only if, $v$ has the same colour of $u$. Let $\varepsilon=1$ if $v$ has the same colour as $u$, otherwise $\varepsilon=0$. As the weight of $(u, v)$ decreases by one and we add the edge $\left(u, v^{\prime}\right)$ of weight 1 in $G^{\prime}$, we get $I_{u}\left(G^{\prime}, w^{\prime}, c^{\prime}\right)=I_{u}(G, w, c)-\varepsilon+w^{\prime}\left(u, v^{\prime}\right) \varepsilon=I_{u}(G, w, c)$. Similarly, $I_{v}\left(G^{\prime}, w^{\prime}, c^{\prime}\right)=I_{v}(G, w, c)$. Finally, $I_{u^{\prime}}\left(G^{\prime}, w^{\prime}, c^{\prime}\right)=I_{v^{\prime}}\left(G^{\prime}, w^{\prime}, c^{\prime}\right)=\varepsilon$. But $I_{u}\left(G^{\prime}, w^{\prime}, c^{\prime}\right) \geq(w(u, v)-1) \varepsilon$ and so $I_{u^{\prime}}\left(G^{\prime}, w^{\prime}, c^{\prime}\right) \leq I_{u}\left(G^{\prime}, w^{\prime}, c^{\prime}\right)$ and $I_{v^{\prime}}\left(G^{\prime}, w^{\prime}, c^{\prime}\right) \leq I_{v}\left(G^{\prime}, w^{\prime}, c^{\prime}\right)$. In summary, we have

$$
\max _{x} I_{x}\left(G^{\prime}, w^{\prime}, c^{\prime}\right)=\max _{x} I_{x}(G, w, c)
$$

and therefore $\omega_{k}(G, w)=\omega_{k}\left(G^{\prime}, w^{\prime}\right)$.

In the worst case, the number of vertices of $G^{*}$ is $n+m\left(w_{\max }-1\right) 2 k$ and the number of edges of $G^{*}$ is $m+m\left(w_{\max }-1\right)[(k+4)(k-1)+2]$ with $n=|V(G)|, m=|E(G)|$ and $w_{\max }=\max _{e \in E(G)} w(e)$.

In conclusion, this construction allows to transform the THRESHOLD IMPROPER COLOURING problem into a problem mixing proper and improper colouring. Therefore the problem consists in finding the minimum $l$ such that a (non-weighted) $l$-improper $k$-colouring of $G^{*}$ exists with the constraint that some subgraphs of $G^{*}$ must admit a proper colouring. The equivalence of the two problems is proved here only for integers weights, but it is possible to adapt the transformation to prove it for rational weights.

\section{Squares of Particular Graphs}

As mentioned in the introduction, WEIGHTED IMPROPER COLOURING is motivated by networks of antennas similar to grids [2]. In these networks, the noise generated by an antenna undergoes an attenuation with the distance it travels.

It is often modelled by a decreasing function of $d$, typically $1 / d^{\alpha}$ or $1 /\left(2^{d-1}\right)$. Here we consider a simplified model where the noise between two neighbouring antennas is normalised to 1 , between antennas at distance two is $1 / 2$ and 0 when the distance is strictly greater than 2 .

Studying this model of interference corresponds to study the WEIGHTED IMPROPER COLOURING of the square of the graph $G$, the graph obtained from $G$ by joining every pair of vertices at distance 2, and to assign weights $w_{2}(e)=1$, if $e \in E(G)$, and $w_{2}(e)=1 / 2$, if $e \in E\left(G^{2}\right)-E(G)$. Observe that in this case the interesting threshold values are the non-negative multiples of $1 / 2$.

In Figure 3 are given some examples of colouring for the square grid. In Figure 3(a) each vertex $x$ has neither a neighbour nor a vertex at distance 2 coloured with its own colour, so $I_{x}\left(G^{2}, w_{2}, c\right)=0$. In Figure 3(b) each vertex $x$ has exactly one vertex of the same colour at distance 2 , so $I_{x}\left(G^{2}, w_{2}, c\right)=1 / 2$.

For any $t \in \mathbb{R}_{+}$, we determine the weighted $t$-improper chromatic number for the square of infinite paths, square grids, hexagonal grids and triangular grids under the interference model $w_{2}$. We also present lower and upper bounds for $\chi_{t}\left(T^{2}, w_{2}\right)$, for any tree $T$ and any threshold $t$. 


\subsection{Infinite paths and trees}

In this section, we characterise the weighted $t$-improper chromatic number of the square of an infinite path, for all positive real $t$. Moreover, we present lower and upper bounds for $\chi_{t}\left(T^{2}, w_{2}\right)$, for a given tree $T$.

Theorem 5. Let $P=(V, E)$ be an infinite path. Then,

$$
\chi_{t}\left(P^{2}, w_{2}\right)= \begin{cases}3, & \text { if } 0 \leq t<1 \\ 2, & \text { if } 1 \leq t<3 \\ 1, & \text { if } 3 \leq t\end{cases}
$$

Proof. Let $V=\left\{v_{i} \mid i \in \mathbb{Z}\right\}$ and $E=\left\{\left(v_{i-1}, v_{i}\right) \mid i \in \mathbb{Z}\right\}$. Each vertex of $P$ has two neighbours and two vertices at distance two. Consequently, the first case $t \geq 3$ is trivial.

There is a 2-colouring $c$ of $\left(P^{2}, w_{2}\right)$ with maximum interference 1 by just colouring $v_{i}$ with colour $i \bmod 2$. So $\chi_{t}\left(P^{2}, w_{2}\right) \leq 2$ if $t \geq 1$. We claim that there is no weighted 0.5 -improper 2-colouring of $\left(P^{2}, w_{2}\right)$. By contradiction, suppose that $c$ is such a colouring. If $c\left(v_{i}\right)=0$, for some $i \in \mathbb{Z}$, then $c\left(v_{i-1}\right)=c\left(v_{i+1}\right)=1$ and $c\left(v_{i-2}\right)=c\left(v_{i+2}\right)=0$. This is a contradiction because $v_{i}$ would have interference 1 .

Finally, the colouring $c\left(v_{i}\right)=i \bmod 3$, for every $i \in \mathbb{Z}$, is a feasible weighted 0 -improper 3-colouring.

Theorem 6. Let $T=(V, E)$ be a tree. Then, $\left\lceil\frac{\Delta(T)-\lfloor t\rfloor}{2 t+1}\right\rceil+1 \leq \chi_{t}\left(T^{2}, w_{2}\right) \leq\left\lceil\frac{\Delta(T)-1}{2 t+1}\right\rceil+2$.

Proof. The lower bound is obtained by two simple observations. First, $\chi_{t}(H, w) \leq \chi_{t}(G, w)$, for any $H \subseteq G$. Let $T$ be a tree and $v$ be a node of maximum degree in $T$. Then, observe that the weighted $t$-improper chromatic number of the subgraph of $T^{2}$ induced by $v$ and its neighbourhood is at least $\left\lceil\frac{\Delta(T)-\lfloor t\rfloor}{2 t+1}\right\rceil+1$. The colour of $v$ can be assigned to at most $\lfloor t\rfloor$ vertices on its neighbourhood. Any other colour used in the neighbourhood of $v$ cannot appear in more than $2 t+1$ vertices because each pair of vertices in the neighbourhood of $v$ is at distance two.

Let us look now at the upper bound. Choose any node $r \in V$ to be its root. Colour $r$ with colour 1 . Then, by a pre-order traversal in the tree, for each visited node $v$ colour all the children of $v$ with the $\left\lceil\frac{\Delta(T)-1}{2 t+1}\right\rceil$ colours different from the ones assigned to $v$ and to its parent. This is a feasible weighted $t$-improper $k$ colouring of $T^{2}$, with $k \leq\left\lceil\frac{\Delta(T)-1}{2 t+1}\right\rceil+2$, since each vertex interferes with at most $2 t$ vertices at distance two which are children of its parent.

\subsection{Grids}

In this section, we show the optimal values of $\chi_{t}\left(G^{2}, w_{2}\right)$, whenever $G$ is an infinite square, or hexagonal or triangular grid, for all the possible values of $t$. The proofs of the theorems presented in this section can be found in the research report [3].

Square Grid. The square grid is the graph in which the vertices are all integer linear combinations $a e_{1}+$ $b e_{2}$ of the two vectors $e_{1}=(1,0)$ and $e_{2}=(0,1)$, for any $a, b \in \mathbb{Z}$. Each vertex $(a, b)$ has four neighbours: its down neighbour $(a-1, b)$, its top neighbour $(a+1, b)$, its right neighbour $(a, b+1)$ and its left neighbour $(a, b-1)$.

Theorem 7. If $G$ is an infinite square grid, then

$$
\chi_{t}\left(G^{2}, w_{2}\right)= \begin{cases}5, & \text { if } t=0 \\ 4, & \text { if } t=0.5 \\ 3, & \text { if } 1 \leq t<3 \\ 2, & \text { if } 3 \leq t<8 \\ 1, & \text { if } 8 \leq t\end{cases}
$$




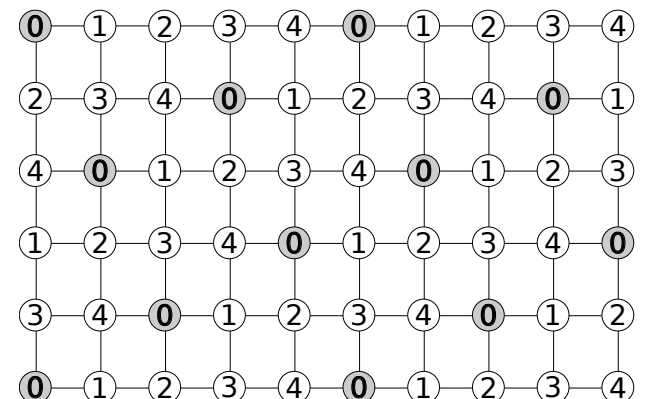

(a)

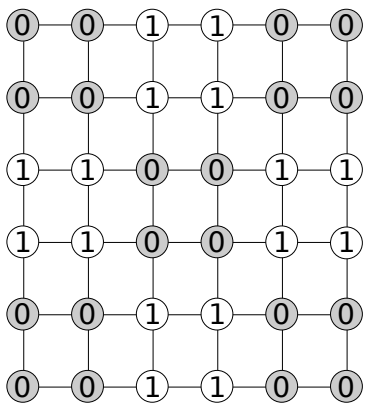

(c)

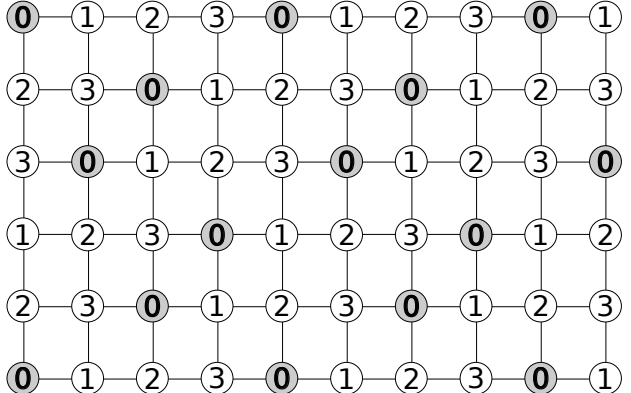

(b)
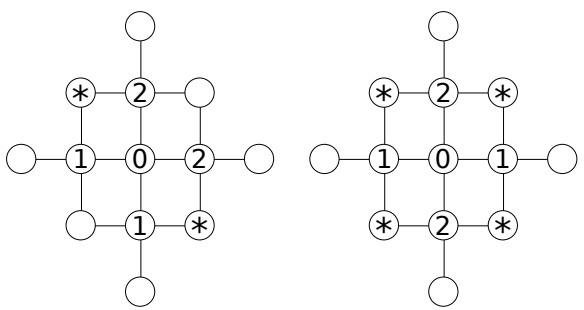

(d)

Fig. 3. Optimal colorings of $G^{2}$, for square grid $G$. Weighted 0-improper 5-colouring of $G^{2}$ in Figure 3(a), weighted 0.5-improper 4-colouring of $G^{2}$ in Figure 3(b) and weighted 3-improper 2-colouring of $G^{2}$ in 3(c). Figure 3(d) shows that there is no weighted 0.5 -improper 3-colouring of $G^{2}$.

Proof. If $t=0$, then the colour of vertex $(a, b)$ must be different from the ones used on its four neighbours. Moreover, all the neighbours have different colours, as each pair of neighbours is at distance two. Consequently, at least 5 colours are needed. Figure 3(a) gives a a weighted 0-improper 5-colouring of $G^{2}$.

When $t=0.5$, we claim that at least four colours are needed to colour $G^{2}$. The proof is by contradiction. Suppose that there exists a weighted 0.5 -improper 3-colouring of it. Let $(a, b)$ be a vertex coloured 0 . No neighbour is coloured 0 , otherwise $(a, b)$ has interference 1 . If three neighbours have the same colour, then each of them will have interference 1 . So two of its neighbours have to be coloured 1 and the two other ones 2 (see Figure 3(d)). Consider now the four nodes $(a-1, b-1),(a-1, b+1),(a+1, b-1)$ and $(a+1, b+1)$. For all configurations, at least two of these 4 vertices have to be coloured 0 . But then $(a, b)$ will have interference at least 1 , a contradiction. A weighted 0.5 -improper 4-colouring of $G^{2}$ is shown in Figure 3(b).

If $t=1$, there exists a weighted 1-improper 3-colouring of $G^{2}$ given by the following construction: for $0 \leq j \leq 2$, let $A_{j}=\left\{(0, j)+a\left(3 e_{2}\right)+b\left(e_{1}+e_{2}\right) \mid \forall a, b \in \mathbb{Z}\right\}$. For $0 \leq j \leq 2$, assign the colour $j$ to all the vertices in $A_{j}$.

Now we prove by contradiction that for $t=2.5$ we still need at least three colours in a weighted 2.5improper colouring of $G^{2}$. Consider a weighted 2.5-improper 2-colouring of $G^{2}$ and let $(a, b)$ be a vertex coloured 0 . Vertex $(a, b)$ has at most two neighbours of colour 0 , otherwise it will have interference 3 . We distinguish three cases:

1. Exactly one of its neighbours is coloured 0 ; let $(a, b-1)$ be this vertex. Then, the three other neighbours are coloured 1. Consider the two set of vertices $\{(a-1, b-1),(a-1, b+1),(a-2, b)\}$ and $\{(a+1, b-$ $1),(a+1, b+1),(a+2, b)\}$; each of them has at least two vertices coloured 0 , otherwise the vertex $(a, b+1)$ or $(a, b-1)$ will have interference 3 . But then $(a, b)$ having 4 vertices at distance 2 coloured 0 has interference 3 , a contradiction.

2. Two neighbours of $(a, b)$ are coloured 0 . 

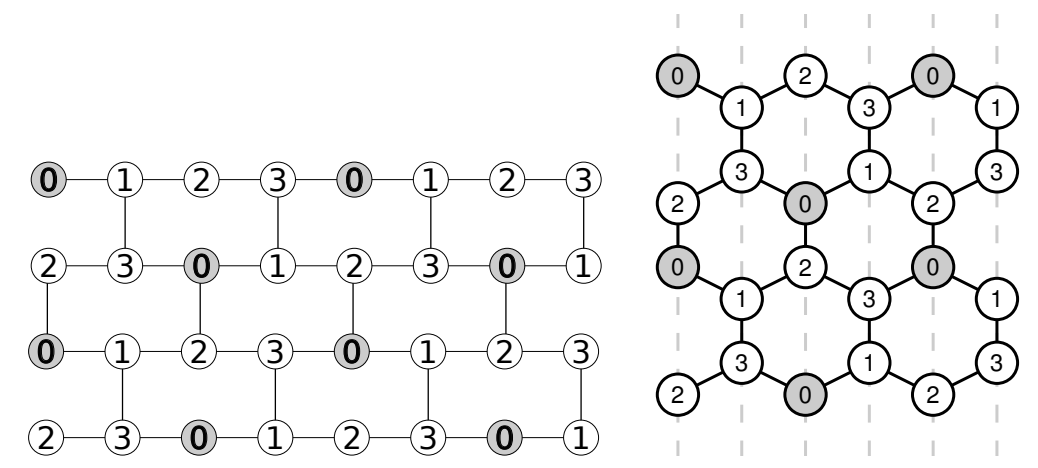

Fig. 4. Optimal construction with $t=0, k=4$. Left: Graph with coordinates. Right: Corresponding hexagonal grid in the euclidean space.

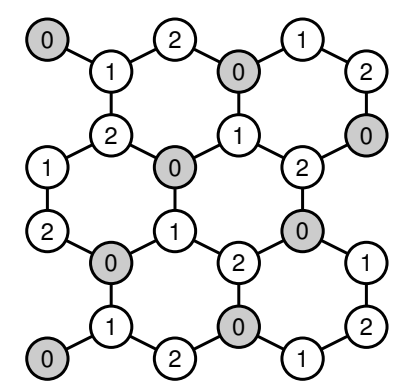

(a) $t=1, k=3$

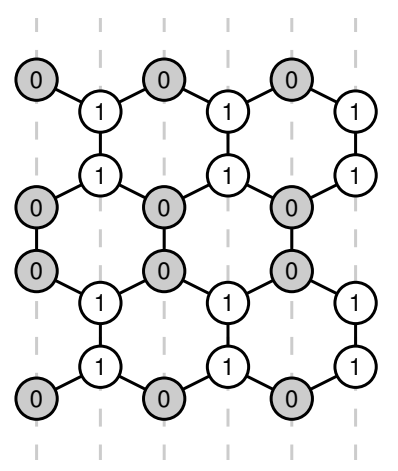

(b) $t=2, k=2$

Fig. 5. Optimal constructions for the hexagonal grid.

(a) These two neighbours are opposite, say $(a, b-1)$ and $(a, b+1)$. Consider again the two sets $\{(a-$ $1, b-1),(a-1, b+1),(a-2, b)\}$ and $\{(a+1, b-1),(a+1, b+1),(a+2, b)\}$; they both contain at least one vertex of colour 0 and therefore $(a, b)$ will have interference 3 , a contradiction.

(b) The two neighbours of colour 0 are of the form $(a, b-1)$ and $(a-1, b)$. Consider the two sets of vertices $\{(a+1, b-1),(a+1, b+1),(a+2, b)\}$ and $\{(a+1, b+1),(a-1, b+1),(a, b+2)\}$; these two sets contain at most one vertex of colour 0 , otherwise $(a, b)$ will have interference 3 . So vertices $(a+1, b-1),(a+2, b),(a, b+2)$ and $(a-1, b+1)$ are of colour 1. Vertex $(a+1, b+1)$ is of colour 0, otherwise $(a+1, b)$ has interference 3. But then $(a, b-2)$ and $(a-1, b-1)$ are of colour 1 , otherwise $(a, b)$ will have interference 3 . Thus, vertex $(a, b-1)$ has exactly one neighbour coloured 0 and we are again in Case 1.

3. All neighbours of $(a, b)$ are coloured 1 . If any of this neighbours has itself a neighbour (distinct from $(a, b)$ ) of colour 1 , we are in case 1 or 2 for this neighbour. Therefore, all vertices at distance two from $(a, b)$ have colour 0 and the interference in $(a, b)$ is 4 , a contradiction.

A weighted 3-improper 2-colouring of $G^{2}$ is given in Figure 3(c). Finally, since each vertex has 4 neighbours and 8 vertices at distance two, there is no weighted 7.5-improper 1-colouring of $G^{2}$ and, whenever $t \geq 8$, one colour suffices.

Hexagonal Grid. To define the hexagonal grid graph, there are many ways to define the system of coordinates. Here, we use grid coordinates as shown in Figure 4. The hexagonal grid graph is then the graph whose vertex set is the pairs of integers $(a, b) \in \mathbb{Z}^{2}$ and where each vertex $(a, b)$ has 3 neighbours: $(a-1, b)$, $(a+1, b)$, and $(a, b+1)$ if $a+b$ is odd, or $(a, b-1)$ otherwise. 


$$
\begin{aligned}
& \text { (0)-1)-(2)-3-4)-(5)-6-(0)-1) } \\
& \text { (2) }-3-(4)-5-6-0-1)-(2-3-4 \\
& \text { (4) }-5-6-0-1-2-3-4-5-6-(0) \\
& \text { (6)-(0)-1 }-(2)-3-(4)-5-6-(0-1)-(2)-3 \\
& \text { (1) }-(2)-3-(4)-5-6-(0-1)-(2-3)-4-5-6
\end{aligned}
$$

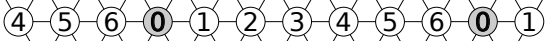

$$
\begin{aligned}
& \text { (0)-1)-(2)-3 - (4)-5)-6-(0)-1)-(2)-3 } \\
& \text { (3)-(4)-5-6-(0-1)-(2)-3-4-(5) } \\
& \text { (6)-0-(1)-(2)-3-(4)-5-6-(0) }
\end{aligned}
$$

(a) Weighted 0-improper 7-colouring of $G^{2}$.

$$
\begin{aligned}
& \text { (4)-(0)-(5)-(2)-(1)-(4)-(3)-(5)-(0) } \\
& \text { (5)-(2)-1)-4-3-5-(0-1)-2-3 } \\
& \text { (1)-4)-3-5)-0-1)-(2)-3-4)-0-(5) } \\
& \text { (3)-5-(0-1)-(2)-3-4)-(0-5-(2)-1)-(4) } \\
& \text { (0)-1)-(2)-3-4)-(0-5)-(2)-1-4)-3-5-(0) } \\
& \text { (3)-4 }-0-(5-2)-1-4-3-5-0-1-2 \\
& \text { (5)-(2)-1)-4 - } 3-5-(0-1)-2-3-(4 \\
& \text { (4) }-3-5-0-1-2-3-4-0-5 \\
& \text { (0)-(1)-(2)-(3)-(4)-(0)-(5)-(2)-1 }
\end{aligned}
$$

(b) Weighted 0.5-improper 6-colouring of $G^{2}$.

$$
\begin{aligned}
& \text { (0)-1)-(3)-(2)-(0)-(1)-(2)-(0) } \\
& \text { (2)-(3)-(0)-(1)-(2)-(0)-(1)-(2)-(3) } \\
& \text { (1)-3)-(2)-(0)- (1)-3)-(2)-(0)-(3)-(2) } \\
& \text { (3)-(0)- (1)-(2)-(3)-(0)- (1)-(2)-(3)-(0)-1)-(2) } \\
& \text { (3)-(2)-(0)-1)-3 - (2)-(0)-1 - (3)-(2)-(0)- (1)-(3) } \\
& \text { (1)-(2)-(3)-(1)-(2)-(0)-(1)-(3)-(0) } \\
& \text { (1)-(3)-(2)-(0)- (1)-(3)-(2)-(0)-(1)-(2) } \\
& \text { (0)-1)-(2)-3-(0)-1-2)-(3)-(0)-1 } \\
& \text { (0)-(1)-(3)-(2)-(1)-(2)-(0) }
\end{aligned}
$$

(c) Weighted 1.5-improper 4-colouring of $G^{2}$.

Fig. 6. Constructions for the triangular grid.

Theorem 8. If $G$ is an infinite hexagonal grid, then

$$
\chi_{t}\left(G^{2}, w_{2}\right)= \begin{cases}4, & \text { if } 0 \leq t<1 \\ 3, & \text { if } 1 \leq t<2 \\ 2, & \text { if } 2 \leq t<6 \\ 1, & \text { if } 6 \leq t\end{cases}
$$

Triangular Grid. The triangular grid is graph whose vertices are all the integer linear combinations $a e_{1}+$ $b e_{2}$ of the two vectors $e_{1}=\left(\frac{\sqrt{3}}{2}, \frac{1}{2}\right)$ and $e_{2}=(0,1)$. Thus we may identify the vertices with the ordered pairs $(a, b)$ of integers. Each vertex $v=(a, b)$ has six neighbours: its left neighbour $(a, b-1)$, its right neighbour $(a, b+1)$, its left-up neighbour $(a+1, b-1)$, its right-up neighbour $(a+1, b+1)$, its left-down neighbour $(a-1, b-1)$ and its right-down neighbour $(a-1, b+1)$.

Theorem 9. If $G$ is an infinite triangular grid, then

$$
\chi_{t}\left(G^{2}, w_{2}\right)= \begin{cases}7, & \text { if } t=0 \\ 6, & \text { if } t=0.5 \\ 5, & \text { if } t=1 \\ 4, & \text { if } 1.5 \leq t<3 \\ 3, & \text { if } 3 \leq t<5 \\ 2, & \text { if } 5 \leq t<12 \\ 1, & \text { if } 12 \leq t\end{cases}
$$

For determining the lower bounds for the cases in which $\chi_{t}\left(G^{2}, w_{2}\right)$ is equal to 2 and 3 , the proofs involved too many subcases to be readable. Then, we used CPLEX with the integer programming formulations we present in Section 4 to validate them. 


\section{Integer Programs, Algorithms and Results}

In this section, we look at how to solve the WEIGHTED IMPROPER COLOURING and THRESHOLD IMPROPER COLOURING for realistic instances. We consider Poisson-Voronoi tesselations as they are good models of antennas networks $[4,8,9]$. We present integer programming models for both problems. Then, we introduce two algorithmic approaches for THRESHOLD IMPROPER COLOURING: a simple greedy heuristic and a Branch-and-Bound algorithm.

\subsection{Integer programs and algorithms}

Integer Programming Models. Given an edge-graph $G=(V, E, w), w: E \rightarrow \mathbb{R}_{+}^{*}$, and a positive real threshold $t$, we model WeIGHTED IMPROPER COLOURING by using two kinds of variables. Variable $x_{i p}$ indicate if vertex $i$ is coloured $p$ and variable $c^{p}$ indicate if colour $p$ is used, for every $1 \leq i \leq n$ and $1 \leq p \leq l$, where $l$ is an upper bound for the number of colours needed in an optimal weighted $t$-improper colouring of $G$ (see Section 2). The model follows:

$$
\begin{aligned}
& \min \quad \sum_{p} c^{p} \\
& \text { subject to } \\
& \sum_{j \neq i} w(i, j) x_{j p} \leq t+M\left(1-x_{i p}\right)(\forall i \in V, \forall p \in\{1, \ldots, l\}) \\
& c^{p} \geq x_{i p} \quad(\forall i \in V, \forall p \in\{1, \ldots, l\}) \\
& \sum_{p} x_{i p}=1 \quad(\forall i \in V) \\
& x_{i p} \in\{0,1\} \quad(\forall i \in V, \forall p \in\{1, \ldots, l\}) \\
& c^{p} \in\{0,1\} \quad(\forall p \in\{1, \ldots, l\})
\end{aligned}
$$

where $M$ is a large integer. For instance, it is sufficient to choose $M>\sum_{(u, v) \in E} w(u, v)$.

For Threshold IMPROPER Colouring, given an edge-weighted graph $G=(V, E, w), w: E \rightarrow \mathbb{R}_{+}^{*}$, and a positive integer $k$, the model we consider is:

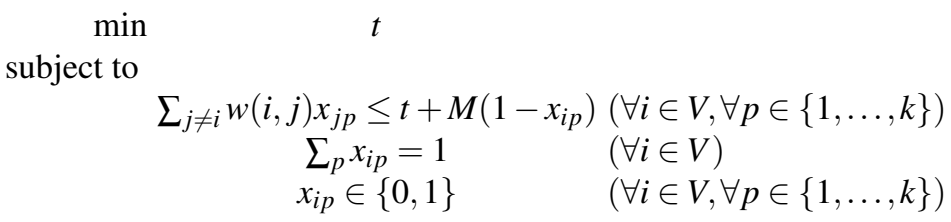

Levelling Heuristic. We develop a heuristic to solve THRESHOLD IMPROPER COLOURING. The idea is to try to level the distribution of interference over the vertices. Each vertex is coloured one after the other by the colour minimising the local interference. More precisely this is achieved by considering for the nodes not yet coloured the "current interference" i.e. the interference induced by the already coloured vertices.

Precisely, consider a vertex $v$ not yet coloured and a colour $i \in\{1, \ldots, k\}$. We define the potential interference $I_{v, i}^{\prime}$ as:

$$
I_{v, i}^{\prime}=\sum_{\left\{u \in N(v) \cap V_{i} \mid c(u)=i\right\}} w(u, v),
$$

where $V_{i}$ is the set of vertices that have already been assigned a colour. The order in which vertices are coloured is decided according to the total potential interference, defined as $I_{v}^{\prime \prime}=\sum_{i=1}^{k} I_{v, i}^{\prime}$. The algorithm finds a feasible colouring in the first step and tries to improve it for $p$ runs, where $p$ is part of the input.

- The interference target is set $t_{t}=M$;

- while the number of runs is smaller than $p$;

- all potential interferences are set to zero;

- while there are still vertices to colour:

* choose a vertex $v$ randomly among the uncoloured vertices that have the maximum total potential interference;

* try each colour $i$ in the order of increasing potential interference $I_{v, i}^{\prime}$ :

- if colouring $v$ with $i$ does not result in interference greater than $t_{t}$ for $v$ or any of its neighbours, colour $v$ with $i$, else try a new colour; 
- if all colours resulted in excessive interferences, start new run.

- If all the vertices were successfully coloured, set $t_{t}=\max _{v \in V, i \in\{1, \ldots, k\}} I_{v}(G, w, c)-\operatorname{gcd}(w)$ and store the colouring as the best found.

As a randomised greedy colouring heuristic, it has to be run multiple times to achieve satisfactory results. This is not a practical issue due to low computational cost of each run. The local immutable colouring decision is taken in time $O(k)$. Then, after each such decision, the interference has to be propagated, which takes time linear in the vertex degree. This gives a computational complexity bound $O(k n \Delta)$.

Branch-and-Bound Algorithm. We also implemented a simple Branch-and-Bound algorithm inspired by the above heuristic. The order in which vertices are coloured is produced by a similar procedure to the one used in the above heuristic. In order to compute this order, we start by marking a random vertex and setting is as the first in a to colour list. Then, as long as there are unmarked vertices, we keep choosing a random vertex $u$ among the unmarked vertices with biggest $\sum_{v \in N(u) \cap V_{m}} w(u, v)$, where $V_{m}$ is the set of already marked vertices. Then we mark $u$ and append it to the to order. A basic Branch-and-Bound colours vertices in the obtained order. Potential interference, as defined for the heuristic, is tracked with the additional step of decreasing the values when backing from a colouring. Colours are tried in the order of increasing potential interference. Thanks to that it produces results similar to the heuristic in a short time. On the other hand it is guaranteed to find the optimal solution in a finite time.

In the following, we compare the performance of these ILP models with the Levelling heuristic and the Branch-and-Bound algorithm .

\subsection{Results}

In this section, we look at the performances of the methods to solve the THRESHOLD IMPROPER COLOURING. We consider Delaunay graphs (dual of Voronoi diagram) for a set of random points. This kind of graph is a natural approximation of a network of irregular cells. The interference model is the one described in Section 3: adjacent nodes interfere by 1 and nodes at distance two interfere by $1 / 2$.

Figure 7 shows a performance comparison of the above-mentioned algorithms. For all the plots, each data point represents an average over ten different graphs. The same graph is used for all values of colours and time limit. Therefore sub-figures 7(b) and 7(c) plot how results for a given problem instance get enhanced with increasing time limits. Plots 7(e) and 7(f) show decreasing interference along increasing the number of colours allowed. Finally plot 7 (d) shows how well all the programs scale with increasing graph sizes.

One immediate observation about both the heuristic and Branch-and-Bound algorithm is that they provide solutions in relatively short time. Despite their naive implementation in a high-level programming language, they tend to find near-optimal results in matter of seconds even for graphs of thousands of vertices. On the other hand, with limited time, they fail to improve up to optimal results, especially with a low number of allowed colours. Although it is easy to envision an implementation faster by orders of magnitude, this may still give little improvement - once a near-optimal solution is found, the Branch-and-Bound algorithm does not improve for a very long time (an example near-optimal solution found in around three minutes was not improved in over six days).

ILP solvers with good Branch-and-Cut implementations do not suffer from this problem. However, they can not take advantage of any specialised knowledge of the problem, only the basic integer programmming representation. Thus it takes much more time to produce first good results. Despite taking advantage of multi-core processing, CPLEX - ILP solver used in this work, does not scale with increasing graph sizes as well as our simple algorithms. Furthermore, Figure 7(e) reveals one problem specific to integer programming. When increasing the number of allowed colours, obtaining small interferences gets easier. But this introduces additional constraints in the linear program, thus increasing the complexity for a solver.

Above observations are valid only for the very particular case of the simple interference function and very sparse graphs. The average degree in Delaunay graph converges to 6. Proposed algorithms also work quite well for denser graphs. Figure 7(f) plots interferences for different numbers of colours allowed found by the programs for an Erdös-Rényi graph with $n=500$ and $p=0.1$. This gives us an average degree of 50 . Both Branch-and-Bound and heuristic programs achieve acceptable, and nearly identical, results. But the large number of constraints makes the linear program nearly inefficient. 


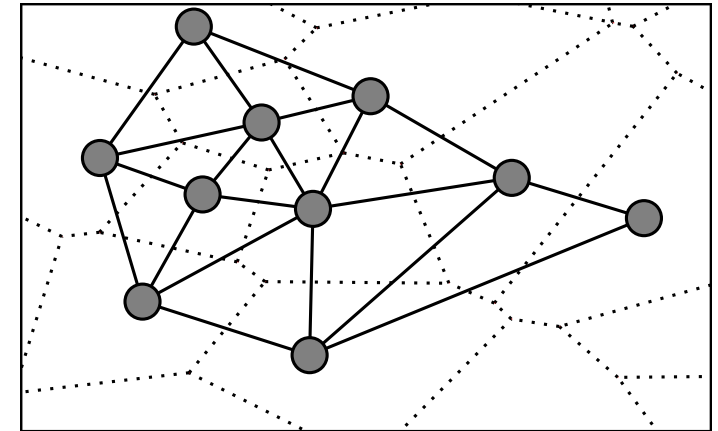

(a) Example Delaunay graph, dotted lines delimit corresponding Voronoi diagram cells

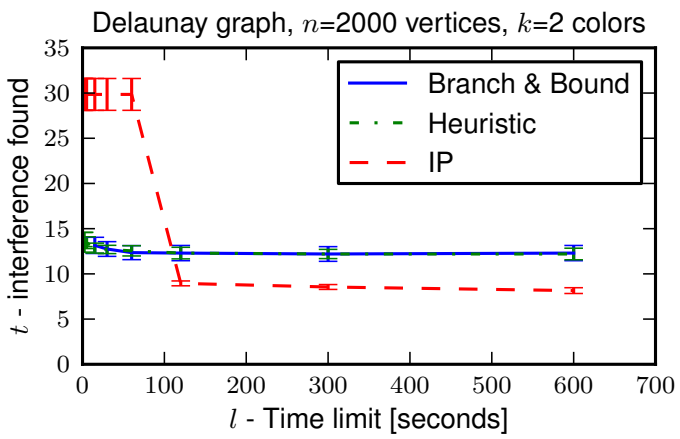

(c) Over time

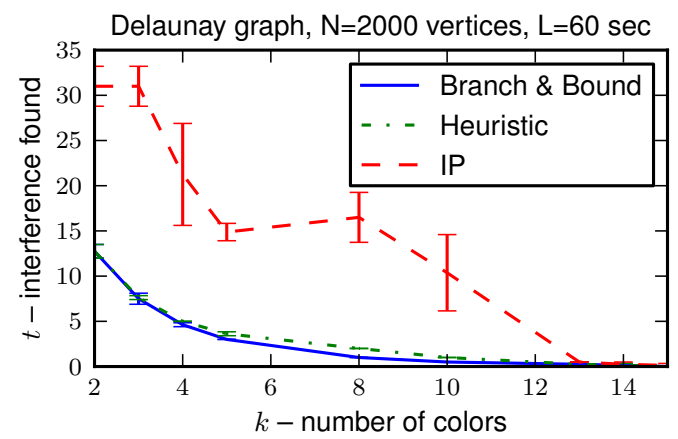

(e) Over colours
Delaunay graph, $n=2000$ vertices, $k=5$ colors

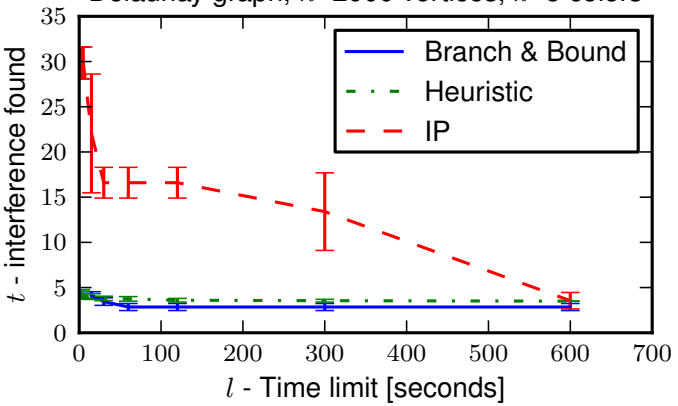

(b) Over time

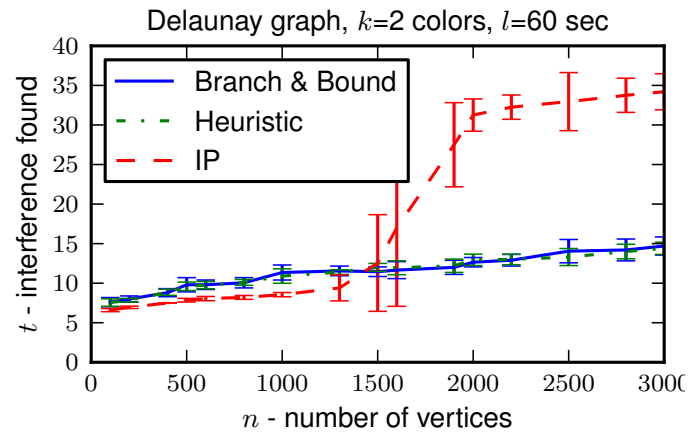

(d) Over size

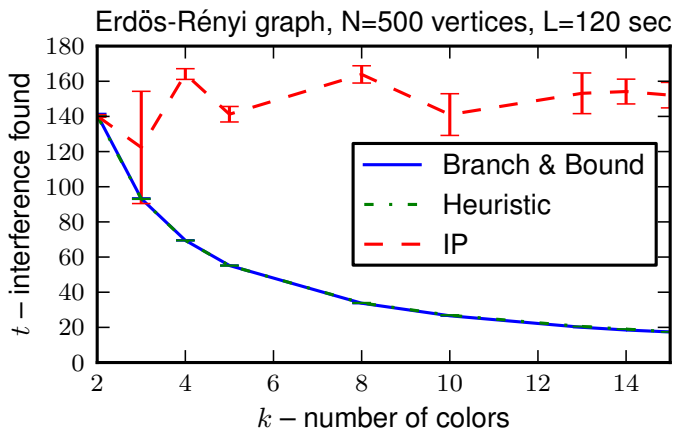

(f) Over colours

Fig. 7. Results comparison for Levelling heuristic, Branch-and-Bound algorithm and Integer Programme.

\section{Conclusion, Open Problems and Future Directions}

In this paper, we introduced and studied a new colouring problem, WEIGHTED IMPROPER COLOURING . This problem is motivated by the design of telecommunication antenna network in which the interferences between two vertices depends on different factors and can take various values. For each vertex, the sum of the interference it receives should be less than a given threshold value.

We first give general bounds on the weighted-improper chromatic number. We then study the particular case of square, triangular and hexagonal grids. For these graphs, we provide their weighted-improper chromatic number for all possible values of $t$. Finally, we propose a heuristic and a Branch-and-Bound algorithm to find good solutions of the problem. We compare their results with the one of an integer program on cell-like networks, Poisson Voronoi tessellations.

Open problems and future directions. Many problems remain to be solved : 
- For the study of the grid graphs, we considered a specific function where vertex at distance one interfere by 1 and vertices at distance 2 by $1 / 2$. Other weight functions should be considered. e.g. $1 / d^{2}$ or $1 /\left(2^{d-1}\right)$, where $d$ is the distance between vertices.

- Other families of graphs could be considered, for example hypercubes.

- Let $G=(V, E, w)$ be an edge-weighted graph where the weights are all equal to 1 or $M$. Let $G_{M}$ be the subgraph of $G$ induced by the edges of weight $M$; is it true that if $\Delta\left(G_{M}\right)<<\Delta(G)$, then $\chi_{t}(G, w) \leq \chi_{t}(G) \leq\left[\frac{\Delta(G, w)+1}{t+1}\right\rceil$ ? A similar result for $L(p, 1)$-labelling [10] suggests it could be true.

\section{References}

1. K.I. Aardal, S.P.M. van Hoesel, A.M.C.A. Koster, C. Mannino, and A. Sassano. Models and solution techniques for frequency assignment problems. Annals of Operations Research, 153(1):79-129, 2007.

2. S. Alouf, E. Altman, J. Galtier, J.F. Lalande, and C. Touati. Quasi-optimal bandwidth allocation for multi-spot MFTDMA satellites. In INFOCOM 2005. 24th Annual Joint Conference of the IEEE Computer and Communications Societies. Proceedings IEEE, volume 1, pages 560-571. IEEE, 2005.

3. J. Araujo, J.-C. Bermond, F. Giroire, F. Havet, D. Mazauric, and R. Modrzejewski. Weighted Improper Colouring. Research Report RR-7590, INRIA, April 2011.

4. F. Baccelli, M. Klein, M. Lebourges, and S. Zuyev. Stochastic geometry and architecture of communication networks. Telecom. Systems, 7(1):209-227, 1997.

5. R. L. Brooks. On colouring the nodes of a network. Mathematical Proceedings of the Cambridge Philosophical Society, 37(02):194-197, 1941.

6. R. Correa, F. Havet, and J-S. Sereni. About a Brooks-type theorem for improper colouring. Australasian Journal of Combinatorics, 43:219-230, 2009.

7. M. Fischetti, C. Lepschy, G. Minerva, G. Romanin-Jacur, and E. Toto. Frequency assignment in mobile radio systems using branch-and-cut techniques. European Journal of Operational Research, 123(2):241-255, 2000.

8. P. Gupta and P.R. Kumar. The capacity of wireless networks. Information Theory, IEEE Transactions on, 46(2):388-404, 2000.

9. M. Haenggi, J.G. Andrews, F. Baccelli, O. Dousse, and M. Franceschetti. Stochastic geometry and random graphs for the analysis and design of wireless networks. Selected Areas in Communications, IEEE Journal on, 27(7):1029-1046, 2009.

10. Frédéric Havet, Bruce Reed, and Jean-Sébastien Sereni. L(2,1)-labelling of graphs. In Proceedings of the nineteenth annual ACM-SIAM symposium on Discrete algorithms, SODA '08, pages 621-630, Philadelphia, PA, USA, 2008. Society for Industrial and Applied Mathematics.

11. R. Karp. Reducibility among combinatorial problems. In R. Miller and J. Thatcher, editors, Complexity of Computer Computations, pages 85-103. Plenum Press, 1972.

12. L. Lovász. On decompositions of graphs. Studia Sci. Math. Hungar., 1:273-238, 1966.

13. C. Mannino and A. Sassano. An enumerative algorithm for the frequency assignment problem. Discrete Applied Mathematics, 129(1):155-169, 2003.

14. D. R. Woodall. Improper colorings of graphs. In R. Nelson and R. J. Wilson, editors, Pitman Res. Notes Math. Ser., volume 218, pages 45-63. Longman Scientific and Technical, 1990.

15. Roger K. Yeh. A survey on labeling graphs with a condition at distance two. Discrete Mathematics, 306(12):1217 $-1231,2006$. 